\title{
The background conditions of the cosmic ray ion charge measurements in the MONICA experiment
}

\author{
Alexey Bakaldin ${ }^{1}$ \\ National Research Nuclear University MEPhI (Moscow Engineering Physics Institute) \\ 31 Kashirskoe shosse, 115409 Moscow, Russia \\ E-mail: bakaldin71@mail.ru
}

\section{Sergey Koldashov}

National Research Nuclear University MEPhI (Moscow Engineering Physics Institute)

31 Kashirskoe shosse, 115409 Moscow, Russia

E-mail: svkoldashovemephi.ru

\section{Sergey Voronov}

National Research Nuclear University MEPhI (Moscow Engineering Physics Institute)

31 Kashirskoe shosse, 115409 Moscow, Russia

E-mail: sergvorlgmail.com

\begin{abstract}
The present contribution is dedicated to the investigation of the background conditions for cosmic ray ion ionization state measurements in MONICA experiment. The future experiment MONICA is aimed to study the cosmic ray ion fluxes from $\mathrm{H}$ till $\mathrm{Ni}$ in energy range 10-300 $\mathrm{MeV} / \mathrm{n}$. The experiment main scientific objective is the measurement of the ion ionization state, as well as elemental, isotope composition and energy spectra of Solar Energetic Particle (SEP), Anomalous (ACR) and Galactic (GCR) cosmic ray fluxes. The observation of the ion fluxes will be carried out with high acceptance multilayer semiconductor telescope-spectrometer MONICA installed onboard satellite. The satellite orbit parameters (circular, altitude is about $600 \mathrm{~km}$, polar) were chosen for the realization of the unique method for the measurement of the charge state of the ions with energies $>10 \mathrm{MeV} / \mathrm{n}$ based on the usage of the Earth magnetic field as a separator of ion charge. To realize the method of the geomagnetic separator it is necessary to detect ions in the Earth magnetosphere at geomagnetic L-shells $\mathrm{L}<6$. At these L-shells the MONICA detectors will be loaded by the trapped energetic particles of the Earth's outer and inner radiation belts. In present work we have carried out the analysis of the background particle fluxes taking into account the recent data of the satellite experiments and known AE-8, AP-8 models and elaborated the recommendations to improve the background conditions for the MONICA experiment.
\end{abstract}

The 34th International Cosmic Ray Conference

30 July- 6 August, 2015

The Hague, The Netherlands

${ }^{1}$ Speaker 


\section{Introduction}

New onboard experiment MONICA [1] is aimed for studying the cosmic ray ion fluxes from $\mathrm{H}$ to $\mathrm{Ni}$ in the energy range $10-300 \mathrm{MeV} / \mathrm{n}$ in the near-Earth space. The main scientific objective of the experiment is to measure ion charge states, elemental and isotope composition and energy spectra of SEP fluxes for the individual Solar events as well as to study the evolution of these characteristics over time. At the same time MONICA will investigate ionization states and isotope compositions of anomalous and galactic cosmic rays as well.

Ion charge states of the SEP observed in the near-Earth space are considered to be sensitive probes for the conditions in acceleration region such as particle acceleration dynamics and efficiency, solar plasma mean electron density and temperature [2], [3]. The ionization states of iron ions are the most informative and sensitive indicator for these processes [4]. As it is known the mean ionization state of iron ions with energies more than $10 \mathrm{MeV} / \mathrm{n}$ is about $\mathrm{Q}_{\mathrm{Fe}} \sim+14$ for "gradual" SEP events and more than $\mathrm{Q}_{\mathrm{Fe}} \sim+20$ for "impulsive" events [5]. Thus, $\mathrm{Fe}$ mean charge state can be used for identification of SEP event type. The mean charges of more light SEP ions $\mathrm{He}, \mathrm{O}, \mathrm{Mg}, \mathrm{Si}, \mathrm{Ar}, \mathrm{Ca}$ with energies $>10 \mathrm{MeV} / \mathrm{n}$ observed during a few "gradual" events are the following: $\mathrm{Q}_{\mathrm{He}} \sim+2, \mathrm{Q}_{\mathrm{O}} \sim+7, \mathrm{Q}_{\mathrm{Mg}} \sim+10, \mathrm{Q}_{\mathrm{Si}} \sim+10.5, \mathrm{Q}_{\mathrm{Ar}} \sim+10, \mathrm{Q}_{\mathrm{Ca}} \sim+11.5$ [4]. The $\mathrm{He}, \mathrm{O}, \mathrm{Mg}, \mathrm{Si}, \mathrm{Ar}$ and $\mathrm{Ca}$ ions with energies more $10 \mathrm{MeV} / \mathrm{n}$ observed during "impulsive" solar events are to be practically totally ionized.

Ion charge composition of the cosmic ray fluxes observed during solar quiet times reflects the features of the processes involved in acceleration and propagation of ACRs and GCRs. As it is known today the ACRs are primarily singly charge ions of the elements with high first ionization potential $(\mathrm{H}, \mathrm{He}, \mathrm{C}, \mathrm{N}, \mathrm{O}, \mathrm{Ne}, \mathrm{Ar}$ and possibly some others) with energies from several up to $50 \mathrm{MeV} / \mathrm{n}$ [6]. It seems that the ACRs are originating in Solar System and represent a sample of neutral interstellar particles that have drifted into the heliosphere, become ionized by the solar wind or UV radiation, and then accelerated up to energies of tens $\mathrm{MeV} / \mathrm{n}$ in the outer heliosphere. In contrast to ACRs, the GCR ions are generated in active processes in our galaxy and should reach the Solar System in essentially fully stripped states.

The investigations will be carried out with the high-acceptance multilayer silicon telescope-spectrometer MONICA [1] installed onboard a satellite which will be launched into the low Earth polar orbit with an altitude about $600 \mathrm{~km}$. The instrument will be pointed to zenith. The spectrometer will detect nuclei in the following energy ranges: $\mathrm{H}$ and $\mathrm{He}-$ from 5 to $70 \mathrm{MeV} / \mathrm{n}$, CNO group - from 10 to $150 \mathrm{MeV} / \mathrm{n}$, Fe group - from 15 to $300 \mathrm{MeV} / \mathrm{n}$. The instrument will measure nuclear charge (Z), mass, energy, and incident angle for each detected particle. The identification of the registered nuclei will be implemented by modified $\Delta \mathrm{E}-\mathrm{E}$ method [7]. The spectrometer acceptance is about $100 \mathrm{~cm}^{2} \mathrm{sr}$, the field of view (FOV) is $\pm 45^{\circ}$.

In the MONICA experiment the method of ion charge (Q) measurement based on using of the Earth magnetic field as a particle charge separator will be implemented. This method is unique for the ions in energy range being investigated. The direct measurement of the charge state of cosmic ray ions at energies more than several $\mathrm{MeV} / \mathrm{n}$ with electrostatic analyzers [8] and any other instrumentations is impossible. The usual $\Delta \mathrm{E}-\mathrm{E}$ spectrometers, used for the registration of the cosmic ray ions, actually measure the nuclei charges of the ions not their ionization state. The ions with the initial charge state $\mathrm{Q}$ and energy $>10 \mathrm{MeV} / \mathrm{n}$ passing through 
spectrometer will be stripped of the orbital electrons within several $\mu \mathrm{g} / \mathrm{cm}^{2}$ of the instrument matter [9].

This contribution is dedicated to study the background conditions for cosmic ray ion charge measurements in MONICA experiment.

\section{Method of the ion charge measurement}

The unique method for the measurement of the ion charge states for energies more than 10 $\mathrm{MeV} / \mathrm{n}$ is using of Earth magnetic field as their separator [4]. It is well known that the penetration depth of ions into the magnetosphere depends on their gyro radius. On a polarorbiting satellite with zenith-pointing charge particle detector onboard moving from a pole to the equator, this can be observed as a flux cut-off for the particles with given magnetic rigidity $\mathrm{R}$ at a corresponding magnetic L-shell $L_{C}$. This effect is depicted in Fig. 1. This figure shows dependence of registration efficiency $E f f(\Delta E)$ for ions detected by the instrument moving from the geomagnetic equator to pole in energy range $\Delta E$ on $\mathrm{L}$-shell. This efficiency is defined as a ratio between the counting rate of the ions detected inside the magnetosphere $N_{\text {in }}$ and the counting rate of the ions detected outside it in interplanetary space $N_{\text {out }}$.

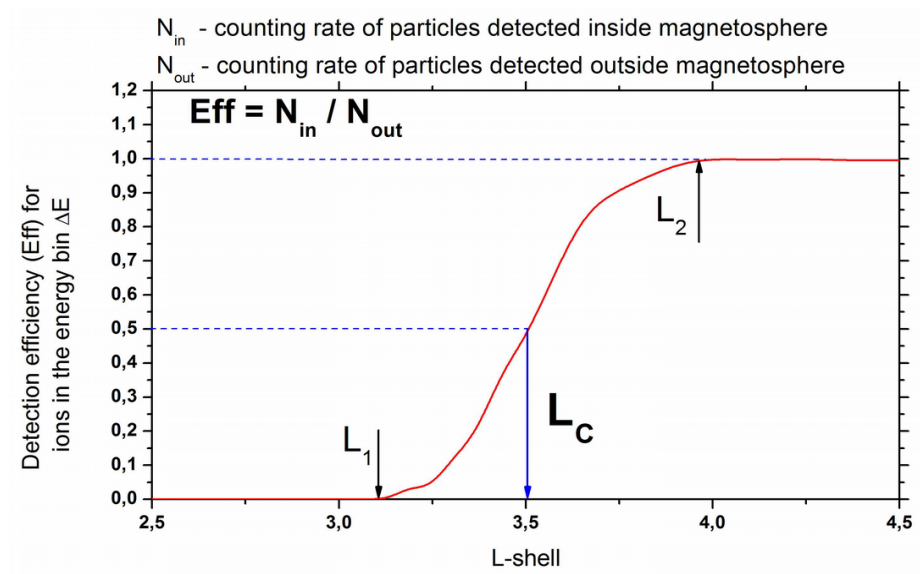

Figure 1: Detection efficiency of the ions registered by instrument versus L-shell. $\mathrm{L}_{1} \div \mathrm{L}_{2}$ - the inflection zone.

As it was assumed in [4], the inverse square of this cut-off L-shell value $L_{C}$ for the real magnetosphere depends linearly on the particle rigidity $R$ :

$$
1 / L c^{2}=a R+b \text {. }
$$

This dependence can be used to determine the mean charge of the particles $Q$ :

$$
\begin{gathered}
Q R=P=\sqrt{(2 \mathrm{M}+E) E}, \\
Q=a \sqrt{(2 \mathrm{M}+E) E} /\left(1 / L c^{2}-b\right),
\end{gathered}
$$

where $E, M=m_{0} c^{2}$ and $P=p c$ are the measured ion kinetic energy, the mass at rest and momentum in energy units $(\mathrm{eV})$ correspondingly.

\section{Region of the separation of the ion charges and the sources of the background}

For measuring of the cosmic ray ion fluxes from $\mathrm{H}$ to $\mathrm{Ni}$ in energy range $10-300 \mathrm{MeV} / \mathrm{n}$ in MONICA experiment it is enough to detect particles in polar caps (at L-shells L $>6$ ) only, i.e. 
outside the Earth radiation belts (RB). The background conditions for the mean ion charge measurement will be absolutely different. In this case the registration of the cosmic ray ions should be carry out at middle and low latitudes where the satellite will cross the outer and inner Earth radiation belts.

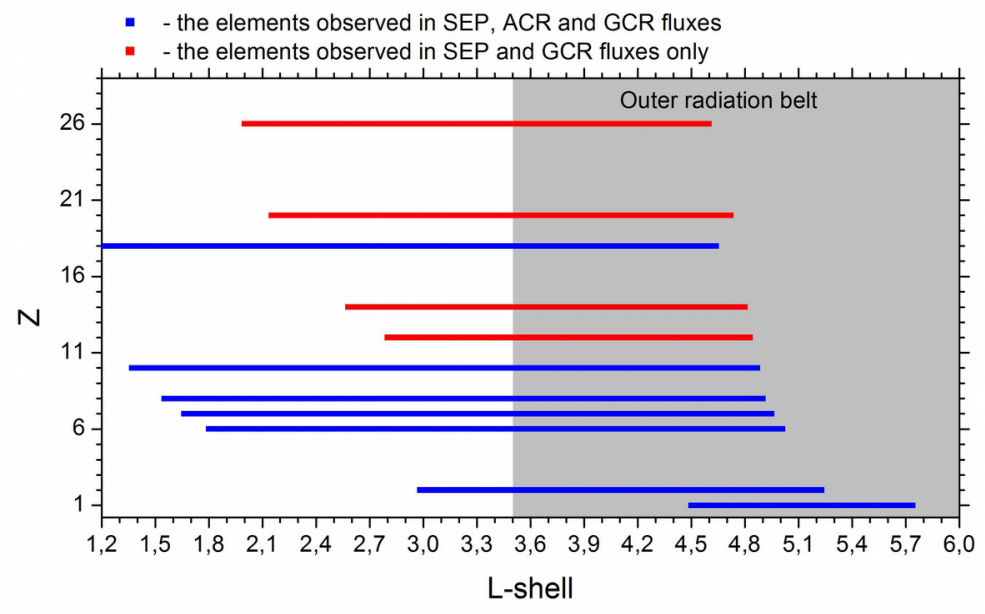

Figure 2: Calculated L-shell intervals $\mathrm{L}_{\min } \div \mathrm{L}_{\max }$, where the inflection zones of the $E f f(L)$ dependences $\mathrm{L}_{1} \div \mathrm{L}_{2}$ will be observed in MONICA experiment, for ions of the different elements from $\mathrm{H}$ to $\mathrm{Fe}$.

In Fig. 2 the evaluated L-shell intervals $\mathrm{L}_{\min } \div \mathrm{L}_{\max }$, where the inflection zones of $\operatorname{Eff}(L)$ dependences $\mathrm{L}_{1} \div \mathrm{L}_{2}$ (see Fig. 1) will be observed in MONICA experiment, for the ions of the different elements from hydrogen to iron is presented. It is seen from the figure, to realize the method of the geomagnetic separator the observation of the ion fluxes should be carried out in the region where $\mathrm{L}<6$.

In Fig. 3 this region of the separation of the ion charges is shown in geographic coordinates. It is localized between $\mathrm{L}=6$ lines. In addition, the experimental map of the counting rates of the ARINA spectrometer top detector obtained during the solar quiet period 1 - 30 September 2006 is presented in Fig. 3 for the estimation of the background conditions in orbit. The ARINA experiment [10] has been carrying out since 15 June 2006 until the present time onboard of the Resurs-DK1 satellite. Its orbit is elliptical and semi-polar one with an inclination $70.4^{\circ}$ and an altitude varying between 350 and $650 \mathrm{~km}$. The top detector of the ARINA spectrometer is plastic scintillator detector with area about $60 \mathrm{~cm}^{2}$ which detects protons with energy $>20 \mathrm{MeV}$ and electrons with energy $>1 \mathrm{MeV}$.

In the MONICA experiment the background main sources in region of the separation of the ion charges (see Fig. 3) are the following: 1) the fluxes of the electrons with energy from some hundred $\mathrm{keV}$ to some $\mathrm{MeV}$ inside zone of the outer $\mathrm{RB}(3.5<\mathrm{L}<6), 2)$ the fluxes of the protons with energy from several $\mathrm{MeV}$ to some hundred $\mathrm{MeV}$ and electrons with energy from some hundred $\mathrm{keV}$ to several tens $\mathrm{MeV}$ inside inner zone. The lower boundaries of the background particle energy ranges are defined by the thickness of the kapton entrance window installed above top detector of the spectrometer, and the upper boundaries correspond to maximum energy of geomagnetically trapped particles in the Earth radiation belts.

The intense fluxes of the high energy protons and electrons of the inner RB crossed by the satellite in the South Atlantic Anomaly (SAA) (see Fig. 3) will penetrate through the MONICA instrument and will cause an overload of all its detectors. 


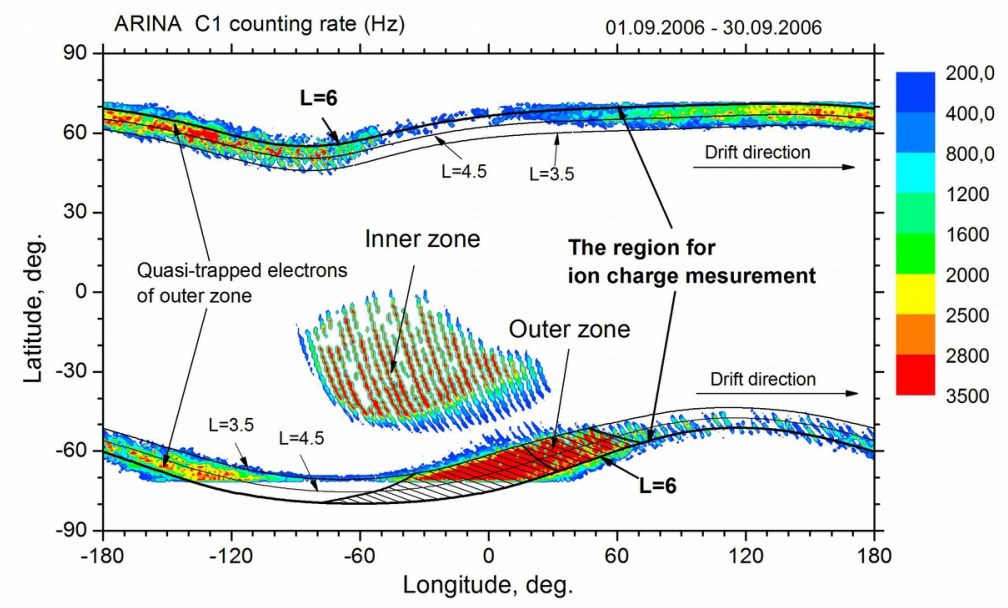

Figure 3: Calculated L-shell intervals $\mathrm{L}_{\min } \div \mathrm{L}_{\max }$, where the inflection zones of the $E f f(L)$ dependences $\mathrm{L}_{1} \div \mathrm{L}_{2}$ will be observed in MONICA experiment, for ions of the different elements from $\mathrm{H}$ to Fe.

As a result the detection of cosmic ray ions in the SAA region will be exceedingly difficult. However, at altitudes about $600 \mathrm{~km}$ the SAA occupies only relatively small region in southern hemisphere inside restricted longitude range from $-90^{\circ}$ to $+30^{\circ}$. Therefore the effective detection of ions outside of the SAA can be carried out at all required L-shells $(L<6)$. The existence of the SAA zone will only lead to a decrease of exposure.

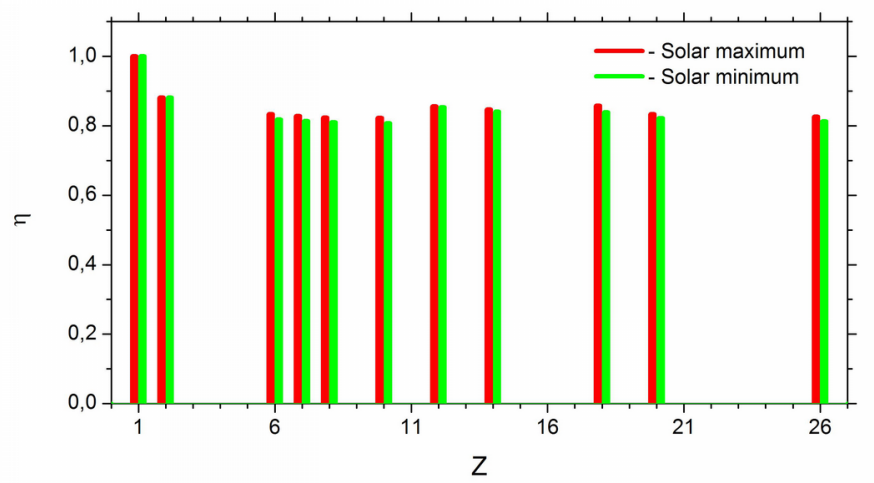

Figure 4: The ratios of the calculated exposures $\eta$ outside and inside the SAA in L-shell interval $\mathrm{L}_{\min } \div \mathrm{L}_{\max }$ for ions of the different elements from $\mathrm{H}$ to Fe for Solar minimum and maximum in MONICA experiment. The evaluations were carried out for the polar circular orbit with inclination $82^{\circ}$ and altitude about $600 \mathrm{~km}$.

In Fig. 4 the ratios of the calculated exposures outside and inside the SAA in L-shell interval $\mathrm{L}_{\min } \div \mathrm{L}_{\max }\left(\eta=T_{\text {Lmin-Lmax }}(\right.$ outside SAA $\left.) / T_{\text {Lmin-Lmax }}\right)$ for ions of the different elements from hydrogen to iron in the MONICA experiment are presented. The evaluations of $\eta$ were implemented for Solar minimum and maximum conditions. The position of the SAA was determined with AP-8 model. One can see from Fig. 4, the rejection of the SAA zone leads to decreasing of the exposure not more than $20 \%$.

Unlike the inner RB the satellite with the MONICA instrument onboard will cross the outer $\mathrm{RB}$ for all longitudes in $\mathrm{L}$-shell range $3.5<\mathrm{L}<6$. As follows from Fig. 2 this range substantially intersects with intervals of the L-shells $\mathrm{L}_{\min } \div \mathrm{L}_{\max }$, where the ion charge measurements will be implemented. So if ion detection will be impossible in outer RB zone this 
will lead to substantial increase of the low boundary of the measured particle magnetic rigidities from $93 \mathrm{MV}$ (corresponding to rigidity of protons with energy $5 \mathrm{MeV}$ ) up to $870 \mathrm{MV}$ (corresponding to vertical cut-off rigidity at $\mathrm{L}=3.5$ ).

The detailed analysis of the possibility for the cosmic ray ion detection in outer RB zone for the MONICA experiment is presented in the next section.

\section{The study of the cosmic ray ion detection conditions in outer zone}

Despite of the fact that there are electrons with energies up to several MeV only in outer $\mathrm{RB}$ and the energy releases for these particles in semiconductor detectors will be much lower than of the detected ions, the multiple superposition of these particle signals can lead to saturation of the instrument electronics. That is why it is very important to carry out the detailed analysis of the background conditions inside zone of the outer $\mathrm{RB}$ at $3.5<\mathrm{L}<6$.

The zone of the outer RB can be divided into two regions (see Fig. 3): 1) the region of the stably trapped particles (it is shown by hatching in Fig. 3); 2) the region of the quasi-trapped particles located inside other part of outer RB zone. Inside the first region electrons are trapped by geomagnetic field doing big number of drift rotations around the Earth during long time. Inside the second region electrons live short time and can do only one incomplete drift rotation after which they are absorbed by the Earth atmosphere during drift from the West to the East near the west boundary of the stable trapped particle region. The quasi-trapped electron fluxes are generated by particle precipitations out of the outer RB. The zone of the quasi-trapped electrons is characterized by high variability of particle fluxes. For example, in the period from 01.09.2006 until 30.09.2006 the changes of these fluxes in several times in the ARINA experiment were observed.

Unfortunately today there are absent the RB models giving the real possibility to fulfill the correct evaluations of the electron fluxes inside outer RB zone for low Earth satellite orbits, especially inside region of the quasi-trapped particles. Therefore the well-known features of the pitch-angle distribution of the geomagnetically trapped particle fluxes were used to estimate the background conditions for ions registration in zone of the outer RB in the MONICA experiment.
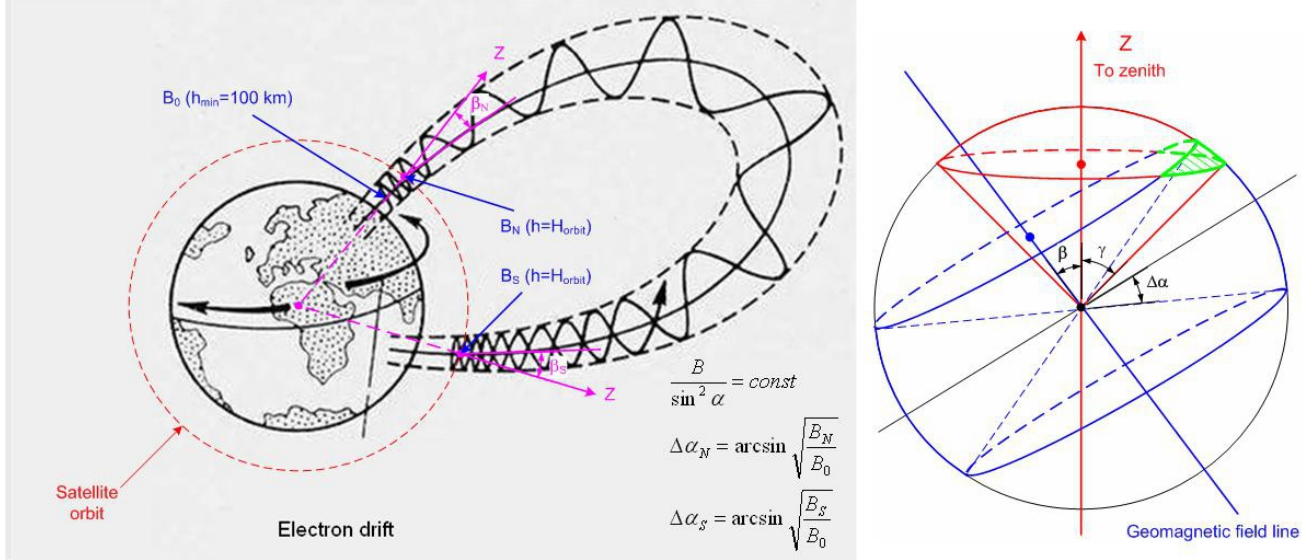

Figure 5: The figure clarifying an algorithm of the evaluation of the instrument FOV contamination fraction inside outer RB.

The trapped electron flux in outer RB is not the omnidirectional. For each point of the RB zone there is strictly fixed range of the pitch-angles $\alpha_{\min } \div \alpha_{\max }$ where the trapped particle flux 
exists. Outside of the range $\alpha_{\min } \div \alpha_{\max }$ this flux is absent. Since the energy of the outer RB electrons is quite low (up to several $\mathrm{MeV}$ ), these particles would be dangerous as a background only in case of their getting into the MONICA spectrometer field of view through the input thin window. The outer RB electrons passing through other elements of the instrument structure will be absorbed inside them and will not reach the sensors.

The fraction of the spectrometer FOV contamination by outer RB electrons for estimation of the background conditions for MONICA experiment was calculated in this work. The calculation of the FOV contamination fraction was realized according an algorithm clarified in Fig. 5. At the first stage the pitch-angle range $\alpha_{\min } \div \alpha_{\max }$ was calculated for given point in outer RB zone with coordinates defined by chosen hemisphere (northern or southern), L-shell and geographic longitude. At the beginning the maximum value of the geomagnetic field $B_{0}$ was determined in mirror points for trapped particles existing in given point of the satellite orbit. The fact was used that for fixes L-shell a particle can be consider quasi-trapped by geomagnetic field if its mirror points in northern and southern hemispheres (with McIlwain coordinates $L, B_{0}$ ) at the observation longitude are located higher then atmosphere boundary $\left(h_{\min } \cong 100 \mathrm{~km}\right)$, i.e. particle can make at least one oscillation between the mirror points. The evaluation of the $B_{0}\left(h_{\min }=100 \mathrm{~km}\right)$ was implemented by means of geomagnetic field line tracing. Then using the adiabatic invariant conservation $B / \sin ^{2} \alpha=$ const (where $B$ is the value of geomagnetic field and $\alpha$ is pitch-angle) the values $\alpha_{\min }$ and $\alpha_{\max }$ were determined using the following expressions:

$$
\alpha_{\text {min }}=\pi / 2-\arcsin \sqrt{B\left(H_{\text {orbit }}\right) / B_{0}\left(h_{\min }\right)}, \quad \alpha_{\max }=\pi / 2+\arcsin \sqrt{B\left(H_{\text {orbit }}\right) / B_{0}\left(h_{\text {min }}\right)},
$$

where $B\left(H_{\text {orbit }}\right)$ is value of the geomagnetic field in studied point (see Fig. 5).
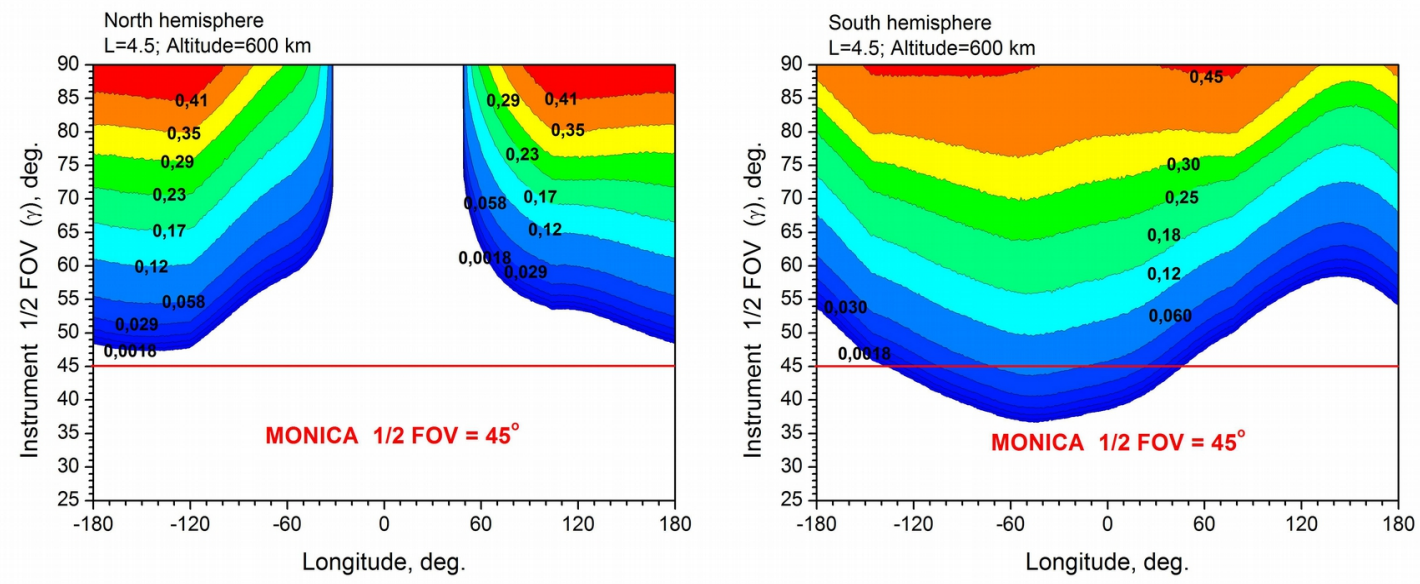

Figure 6: The calculated dependences of the instrument FOV contamination fraction $\xi$ by trapped particles in outer RB when satellite orbit at altitude $600 \mathrm{~km}$ crossing the L-shell $\mathrm{L}=4.5$ on the FOV value and geographical longitude of $\mathrm{L}=4.5$ crossing for northern (left) and southern (right) hemispheres.

At the second stage the value of the spatial angle $\Delta \Omega$ was determined which corresponds to directions of outer RB particles arriving into instrument FOV. Then the fraction of the FOV contamination $\xi$ was calculated as ratio of $\Delta \Omega$ to whole instrument FOV $\Omega_{\mathrm{FOV}}$ : $\xi=\Delta \Omega / \Omega_{\mathrm{FOV}}$. The determining of spatial angle $\Delta \Omega$ is clarified in Fig. 5 where $\Delta \Omega$ is highlighted by green color. The spatial angle $\Delta \Omega$ corresponds to the area of the crossing the FOV (red spherical sector with angle $\gamma=1 / 2$ FOV in Fig. 5) and the area of the feasible trapped particle directions (blue 
spherical segment with angle $\Delta \alpha=\left(\alpha_{\max }-\alpha_{\min }\right) / 2$ in Fig. 5 which axis is the direction of geomagnetic field).

In Fig. 6 the calculated dependences of the instrument FOV contamination fraction $\xi$ by outer RB particles when satellite orbit at altitude $600 \mathrm{~km}$ crossing the L-shell L=4.5 (where intensity of the outer RB particles has maximum value) on the FOV value and geographical longitude of $\mathrm{L}=4.5$ crossing are presented for northern (left) and southern (right) hemispheres. As it is follows from Fig. 6 the contamination of the MONICA instrument FOV by outer RB electrons is observed in southern hemisphere only in longitude range from $-120^{\circ}$ to $+50^{\circ}$ in the region of the stably trapped particles (see Fig. 3). The contamination fraction value $\xi$ does not exceed $6 \%$.

Thus the trapped electron flux in outer RB zone practically will not affect functioning of the MONICA detectors excluding may be the top semiconductor detector D1 [1] only which can be protected additionally with collimator.

\section{Conclusions}

The detailed analysis of the background conditions for cosmic ray ion charge measurements for satellite experiment MONICA was presented in this work.

It was shown that for chosen satellite orbit parameters (circular, polar with inclination $\geq 82^{\circ}$ and altitude about $600 \mathrm{~km}$ ) the RB particle background fluxes will not have the significant impact on results of the MONICA experiment. Cosmic ray ion registration especially light ones will be difficult inside SAA zone because of the instrument detector overloads. However, the excluding of this region out of the observations will lead to decreasing of the exposure and detected particle statistics inside the regions of the ion charge measurements not more than $20 \%$. The outer RB electron fluxes will not in general influence on the MONICA instrument detector functioning. The top semiconductor detector can be shielded against outer RB electrons additionally with collimator.

This work was supported by the RFBR, grant 13-02-00477.

\section{References}

[1] A. Bakaldin, et al., Proc. 31st ICRC, http://icrc2009.uni.lodz.pl/proc/pdf/icrc0387.pdf (2009).

[2] V. Ostryakov, M. Stovpyuk, Solar Physics 189, 2357 (1999).

[3] Y. Kartavykh, V. Ostryakov, 26th ICRC 6, 272 (1999).

[4] M. Oetliker, et al., Ap.J. 477, 495 (1997).

[5] D. Reames, SpaceSci.Rev. 90, 413 (1999).

[6] R. A. Mewaldt, R. S. Selesnick, and J. R. Cummings, Geophysical Monograph 97, 35 (1996).

[7] A. Bakaldin, et al., Instruments and Experimental Techniques 53, 4490 (2010).

[8] E. Mobius, et al., SpaceSci.Rev. 86, 447 (1998).

[9] V. Shevelko, et al., J. Phys. B: At. Mol. Opt. Phys. 42,065202 (2009).

[10] A. V. Bakaldin, et al., Cosmic Research 45, 5445 (2007). 